\title{
Upregulation of miR-23b Enhances the Autologous Therapeutic Potential for Degenerative Arthritis by Targeting PRKACB in Synovial Fluid-Derived Mesenchymal Stem Cells from Patients
}

\author{
Onju Ham ${ }^{2,3,10}$, Chang Youn Lee ${ }^{4,10}$, Byeong-Wook Song ${ }^{2,3}$, Se-Yeon Lee ${ }^{2,3}$, Ran Kim ${ }^{1}$, Jun-Hee Park ${ }^{4}$, \\ Jiyun Lee ${ }^{2,3}$, Hyang-Hee Seo ${ }^{2,3}$, Chae Yoon Lee ${ }^{1}$, Yong-An Chung ${ }^{5}$, Lee-So Maeng ${ }^{5}$, Min Young Lee ${ }^{6}$, \\ Jongmin $\mathrm{Kim}^{7}$, Jihwan Hwang ${ }^{8}$, Dong Kyun Woo', and Woochul Chang ${ }^{1, *}$
}

The use of synovial fluid-derived mesenchymal stem cells (SFMSCs) obtained from patients with degenerative arthropathy may serve as an alternative therapeutic strategy in osteoarthritis (OA) and rheumatoid arthritis (RA). For treatment of OA and RA patients, autologous transplantation of differentiated MSCs has several beneficial effects for cartilage regeneration including immunomodulatory activity. In this study, we induced chondrogenic differentiation of SFMSCs by inhibiting protein kinase A (PKA) with a small molecule and microRNA (miRNA). Chondrogenic differentiation was confirmed by PCR and immunocytochemistry using probes specific for aggrecan, the major cartilaginous proteoglycan gene. Absorbance of alcian blue stain to detect chondrogenic differentiation was increased in $\mathrm{H}-89$ and/or miRNA-23btransfected cells. Furthermore, expression of matrix metalloproteinase (MMP)-9 and MMP-2 was decreased in treated

\footnotetext{
${ }^{1}$ Department of Biology Education, College of Education, Pusan National University, Busan 609-735, Korea, ${ }^{2}$ Cardiovascular Research Institute, ${ }^{3}$ Brain Korea 21 PLUS Project for Medical Science, Yonsei University College of Medicine, Seoul 120-752, Korea, ${ }^{4}$ Department of Integrated Omics for Biomedical Sciences, Graduate School, Yonsei University, Seoul 120-759, Korea, ${ }^{5}$ Institute of Catholic Integrative Medicine, Incheon St. Mary's Hospital, The Catholic University of Korea College of Medicine, Incheon 403-720, Korea, ${ }^{6}$ Department of Molecular Physiology, College of Pharmacy, Kyungpook National University, Daegu 702701, Korea, ${ }^{7}$ Department of Life Systems, Sookmyung Women's University, Seoul 140-742, Korea, ${ }^{8}$ Department of Microbiology, College of Natural Science, Pusan National University, Busan 609-735, Korea, ${ }^{9}$ College of Pharmacy and Research Institute of Pharmaceutical Sciences, Gyeongsang National University, Jinju 660-701, Korea, ${ }^{10}$ These authors contributed equally to this work.

*Correspondence: wchang1975@pusan.ac.kr
}

Received 3 February, 2014; revised 21 May, 2014; accepted 22 May, 2014; published online 11 June, 2014

Keywords: autologous cell transplantation, microRNAs, small molecules, synovial fluid-derived mesenchymal stem cells cells. Therefore, differentiation of SFMSCs into chondrocytes through inhibition of PKA signaling may be a therapeutic option for OA or RA patients.

\section{INTRODUCTION}

Major restrictions to therapy of damaged adult cartilage include the attenuated intrinsic self-healing capacity and a low abundance of regenerated chondrocytes in sites of injured cartilage (Yamaoka et al., 2006). To overcome these problems during cartilage regeneration and repair, several techniques including cell-therapy, gene therapy, and protein therapy have been proposed (Zhai et al., 2001).

Mesenchymal stem cells (MSCs) are present in various adult tissues and differentiate into various cell types of connective tissue lineage including bone, fat, muscle, and cartilage (Andersen et al., 2011; Barry and Murphy et al., 2004). Due to this multi-potential capacity, MSCs can play a key role in the maintenance of homeostasis in bone and cartilage. Thus, MSCs are considered as strong candidates for cell-based therapies for regeneration or replacement of not only damaged skeletal tissues, but also soft tissue organs (De Ugarte et al., 2003; Friedenstein, 1980; Horwitz et al.,1999; Prockop et al., 2000). Among numerous studies related to MSCs in adult tissues, many have been concerned with synovial fluid-derived mesenchymal stem cells (SFMSCs) obtained from patients with primary knee osteoarthritis (OA) (Jones et al., 2010; Lee et al., 2012; Sakaguchi et al., 2005; Sekiya et al., 2012). Compared to MSCs produced from other tissues, SFMSCs have a higher chondrogenic capacity and can be harvested easily during arthrocentesis or routine arthroscopic examination without damaging normal tissue (Iwata et al., 1993; Nishimura et al., 1999). A study reported that SF derived mesenchymal progenitor cells (SF MPCs) reduce the inflammatory intra-articular environment in rheumatoid arthritis (RA) joints (Jones et al., 2004). Chondrocyte differentiation involves MSC condensation, recruitment, and migration, followed by differentiation and maturation into cartilage (Goldring et al., 2006; Olsen et al., 2000). 
Different protein kinases regulate complex cellular processes, and thus chemical compounds that target specific protein kinases can be used to alter cellular functions such as differentiation into various cell types (Cohen, 1999; Davies et al., 2000). Along these lines, we previously reported that modulation of the activity of protein kinases causes recognizable changes in the specific differentiation rates of MSCs based on the ability of screening inhibitors and activators of major protein kinase subfamilies to alter the orchestration of multiple signaling pathways (Hwang et al., 2008).

MicroRNAs (miRNAs) are a class of non-coding RNAs ( 22 nt in length) that play a key role in controlling gene expression by post-transcriptional regulation of target mRNAs or translational inhibition of target protein by associating with the 3 'untranslated region ( $3^{\prime}$ UTR) of target genes (Jin et al., 2012). Importantly, miRNAs can regulate a wide range of cellular processes including cell proliferation, apoptosis, embryonic development, and differentiation (Bagga et al., 2005; Giraldez et al., 2006; Wu et al., 2006; Xiong et al., 2013). Only a few studies have evaluated miRNA expression during chondrogenic differentiation in MSCs (Kobayashi et al., 2008; Nakamura et al., 2011; Yan et al., 2011). Thus, specific miRNAs and the associated target genes that regulate chondrocyte differentiation have not been well established.

Here, we identified the therapeutic possibility of SFMSCs by inducing their differentiation into chondrocytes. We found that $\mathrm{H}-89$ and miRNA-23b facilitated differentiation of SFMSCs into chondrocytes. These differentiated stem cells may be an important tool for autologous stem cell therapy in repairing damaged cartilage.

\section{MATERIALS AND METHODS}

Isolation of SFMSCs from patients with OA

Synovial fluid samples were obtained from 10 osteoarthritis patients by joint puncture. SFMSCs were isolated from synovial fluid within $4 \mathrm{~h}$ of aspiration. The samples were washed with phosphate buffered saline (PBS) with $0.1 \mathrm{M}$ EDTA for $10 \mathrm{~min}$ at $x 363 \mathrm{~g}$ The cells were then collected and suspended in DMEM containing $10 \%$ fetal bovine serum (FBS, Hyclone, USA) and incubated in $37^{\circ} \mathrm{C}$ with $5 \% \mathrm{CO}_{2}$. After 3 days, the medium was replaced to remove non-adherent cells. Ethical approval for the use of SFMSCs was obtained from the Institutional Review Board of Catholic University Medical center.

Cultures of synovial fluid cells, bone marrow-derived mesenchymal stem cells (MSCs), and HeLa cells

Synovial fluid was diluted PBS, filtered through a $70 \mathrm{~mm}$ nylon filter (Becton Dickinson, USA) to remove debris, and plated in $60 \mathrm{~cm}^{2}$ culture dishes (Nalgene Nunc International, USA) in complete culture medium consisting of modified essential medium (a-MEM; Invitrogen, USA) supplemented with $10 \%$ fetal bovine serum (Invitrogen), $100 \mathrm{U} / \mathrm{ml}$ penicillin, $100 \mathrm{mg} / \mathrm{ml}$ streptomycin, and $250 \mathrm{ng} / \mathrm{ml}$ amphotericin B (Invitrogen). Cells were incubated at $37^{\circ} \mathrm{C}$ in a $5 \%$ humidified $\mathrm{CO}_{2}$ atmosphere. Human bone marrow derived MSCs (BMMSCs, Cat no: PT-2501) were purchased from Lonza (USA). BMMSCs were maintained according to the manufacturer's instruction. We used passage 5-7 hMSCs for differentiation. The HeLa (human epithelial carcinoma) cell line was purchased from the ATCC (USA) and cultured according to the ATCC guidelines.

Chondrocyte differentiation

For inducing differentiation of MSCs into chondrocytes, cells were plated on culture dish or plate. Cells were plated at $\sim 60 \%$ confluence in $10 \%$ FBS contained DMEM. $1 \mu \mathrm{M} \mathrm{H}-89$ was treated onto cells every 3 days and $100 \mathrm{nM}$ miR-23b mimic was transfected into cells every 7 days. Cells were incubated for 11 days while the culture media were replaced every 3 days. After 11 days, chondrogenic differentiated cells were collected.

Real-time polymerase chain reaction (PCR)

RNA was extracted using $500 \mu$ l of Trizol ${ }^{\circledR}$ reagent (Sigma, USA). Total cellular RNA was extracted using chloroform, isopropanol, and a DEPC-treated $75 \%$ ethanol procedure. RNA was re-suspended in $40 \mu \mathrm{l}$ nuclease-free water. The quantity and quality of the isolated RNA was determined by calculating the OD260/OD280 ratio with a DU 640 spectrophotometer (Eppendorf, Germany). The threshold cycle (Ct) of each target gene was defined automatically in the linear PCR amplification phase and normalized to the cycle number of the U6 control $\left({ }^{\Delta} \mathrm{Ct}\right.$ value). The relative difference in expression levels of each miRNA was calculated $\left({ }^{\Delta \Lambda} \mathrm{Ct}\right)$ and reported as fold induction $\left(2^{-}\right.$ ${ }^{\triangle \Delta} \mathrm{Ct}$ ). The sequences of hsa-miRNA primers were: miRNA-23b: 5'-AUCACAUUGCCAGGGAUUACC-3', miRNA-26a: 5'UUCAAGUAAUCCAGGAUAGGCU-3', miRNA-133a: 5'UUUGGUCCCCUUCAACCAGCUG-3', miRNA-181a: 5'-AACAUUCAACGCUGUCGGUGAGU-3' and miRNA-221: 5'-AGCUACAUUGUCUGCUGGGUUUC-3'. Total synthesized CDNA was used for real-time PCR, which was detected with SyberGreen dye. In addition, aliquots of the RT reaction mixture were subjected to PCR for following primer set: GAPDH, 5'-CATGGGTGTGAACCATGAGAA-3 and 5'-GGTCATGAGTCCTTCCACGAT-3 (133 bp); Aggrecan, 5'-TTCAGTGGCCTACCAAGTGGCATA-3' and 5'-AGCCTGGGTTACAGATTCCACCAA -3' (165 bp); collagen type II, 5'-TTTCCCAGGTCAAGATGGTC-3' and 5'-CTGCAGCACCTGTCTCACCA-3' (377 bp); Sox9, 5'-ATCTGAAGAAGGAGAGCGAG-3' and 5'TCAGAAGTCTCCAGAGCTTG-3' (264bp), collagen type X, 5'-ATGACCCAAGGACTGGAATCTTTA-3' and 5'-CTGAGAAAGAGGAGTGGACATAC-3' (276 bp). PCR oligonucleotides and reagents used for reverse transcription were obtained from Roche (Germany). PCR conditions for real time analysis of aggrecan and miRNAs were $95^{\circ} \mathrm{C}$ for $10 \mathrm{~min}$, followed by 45 cycles of denaturation at $95^{\circ} \mathrm{C}$ for $10 \mathrm{~s}, 60^{\circ} \mathrm{C}$ for $35 \mathrm{~s}, 72^{\circ} \mathrm{C}$ for $1 \mathrm{~s}$, with a final extension step of $40^{\circ} \mathrm{C}$ for $10 \mathrm{~s}$. For real-time PCR, $500 \mathrm{ng}$ of the cDNA from the reverse transcription reaction was used. PCR reactions consisted of LightCycler® 480 Probes Master mix and $10 \mathrm{nM}$ each of the forward and reverse primers in a total volume of 20 $\mu \mathrm{l}$.

\section{Alcian blue staining}

Cells were washed first with PBS (Gibco, USA) three times and fixed with $10 \%$ formaldehyde (Sigma) for $10 \mathrm{~min}$ at room temperature. Staining was accomplished by applying a solution of $1 \%$ alcian blue $8 \mathrm{GX}$ (Bio Basic Canada Inc., Canada) in $0.1 \mathrm{M}$ $\mathrm{HCl}(\mathrm{pH} 1.0$, Sigma) to the cells for $30 \mathrm{~min}$ at room temperature. To quantify the intensity of staining, the stained culture plates were rinsed with PBS three times and the optical density of extracted dye was measured at $650 \mathrm{~nm}$.

\section{microRNA transfection}

MiRNA and scrambled RNA oligomers (NC) were purchased from Genolution Pharmaceuticals (Genolution Inc., Korea). The reagents were transfected into hMSCs at a final concentration of $100 \mathrm{nM}$ using siLentFect ${ }^{\mathrm{TM}}$ Lipid reagent (Bio-Rad). miRNA mimics and scrambled RNA oligomers were added to cells according to the manufacturer's instructions. After a $4 \mathrm{~h}$ transfection, the media was replaced. 

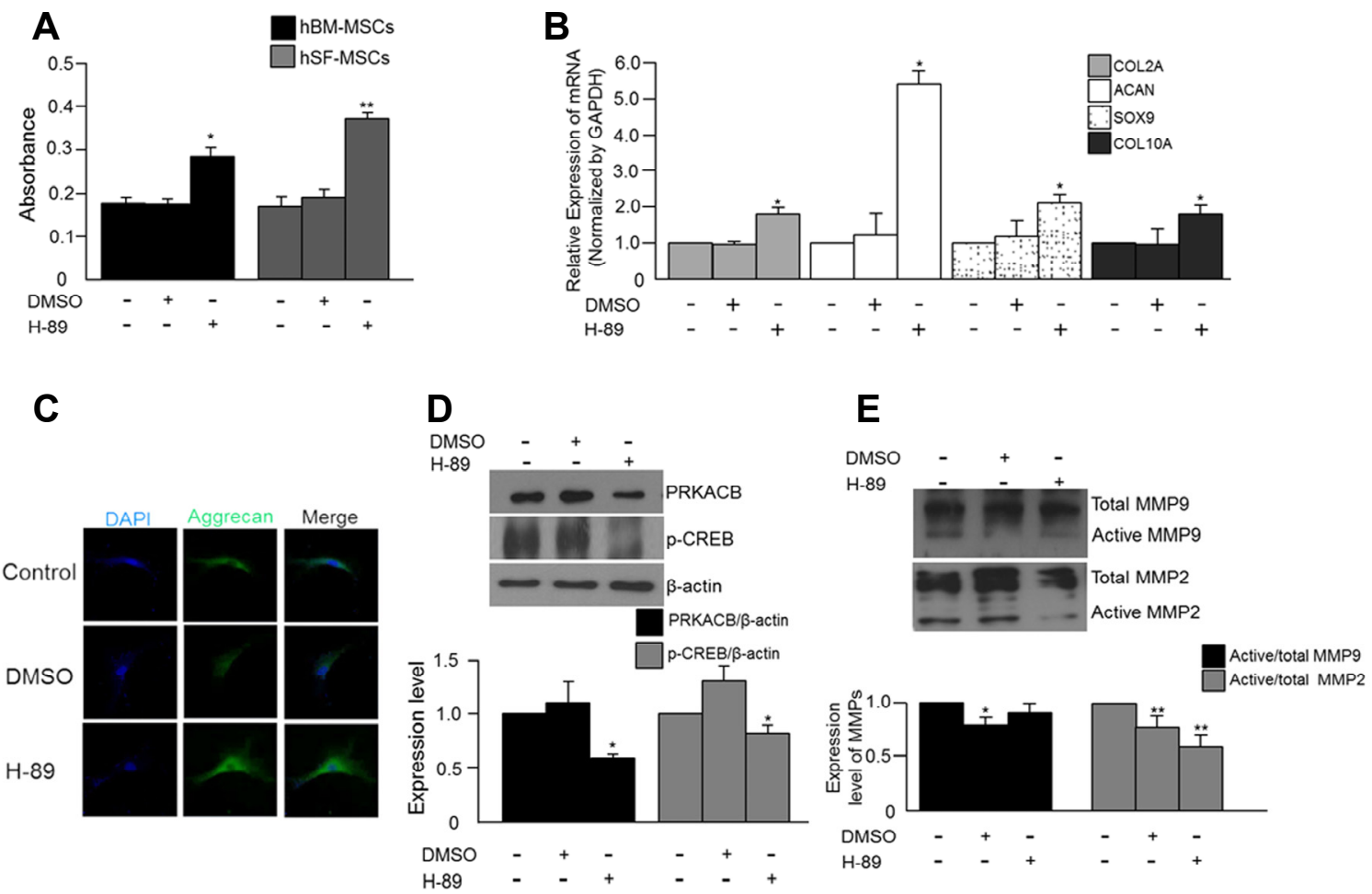

Fig. 1. Effect of H-89 on differentiation of SFMSCs into chondrocytes (A) Absorbance of alcian blue was detected at $650 \mathrm{~nm}$ in BMMSCs and SFMSCs. (B) Expression of aggrecan, collagen type II, collagen type X, and Sox9 in SFMSCs was measured by real-time PCR. (C) Expression of aggrecan was shown by ICC. (D) Expression of PRKACB and p-CREB in SFMSCs was detected by Western blot. (E) Expression of MMP-9 and MMP-2 in H-89-treated cells ( ${ }^{*} p<0.05$ vs control; ${ }^{* *} p<0.001$ vs control).

\section{Western blot analysis}

SFMSCs were collected in lysis buffer containing $1 \%$ Triton $\mathrm{X}$ 100 , protease inhibitor cocktail, and $1 \mathrm{mM}$ phenylmethylsulfonyl fluoride (PMSF) and incubated at $4^{\circ} \mathrm{C}$ for $30 \mathrm{~min}$. Equal quantities of proteins were then subjected to $10 \%$ sodium dodecyl sulfate-polyacrylamide gel electrophoresis (SDS-PAGE). Next, proteins were transferred to polyvinylidene difluoride membranes (Millipore, USA) at $100 \mathrm{~V}$ and $135 \mathrm{~mA}$ for $1 \mathrm{~h}$. The membranes were then blocked with Tris-buffered saline-Tween 20 (TBS-T, 0.05\% Tween 20) and 5\% skim milk for $1 \mathrm{~h}$ at room temperature or overnight $(\mathrm{O} / \mathrm{N})$ at $4^{\circ} \mathrm{C}$ then treated with the primary antibodies anti-p-CREB, anti-matrix metalloproteinase(MMP)-2, anti-MMP-9, anti-PRKACB, or anti-beta actin for 1 $h$ at room temperature or $\mathrm{O} / \mathrm{N}$ at $4^{\circ} \mathrm{C}$. Polyclonal anti $\mathrm{p}$-CREB antibody was obtained from Cell signaling Technology (USA); anti-beta-actin antibody was obtained from Sigma; anti MMP-9 antibody was obtained from Abcam (UK); and the monoclonal PRKACB antibody and the polyclonal MMP-2 antibody were obtained from Santa Cruz Biotechnology (USA). Membranes were washed with TBS-T $(0.05 \%$ Tween 20$)$ three times at room temperature and incubated with a horseradish peroxidase-conjugated secondary antibody. After washing the membrane six times, immunoreactive proteins were detected using an ECL system (Amersham Biosciences, Japan). Anti-mouse or anti-rabbit horse-radish peroxidase-conjugated secondary antibodies were obtained from Santa Cruz Biotechnology. Image $\mathrm{J}$ software was used for quantification.
Immunocytochemistry

Cells were seeded on four-well plastic dishes to induce differentiation, washed twice with PBS, and then fixed with $4 \%$ formaldehyde in $0.5 \mathrm{ml}$ of PBS for $30 \mathrm{~min}$ at room temperature. The cells were then washed again with PBS, blocked with PBS containing $0.5 \% \mathrm{BSA}$, and then incubated for $1 \mathrm{~h}$ with primary antibodies including aggrecan, CD34, CD44, CD73, and CD90. The cells were then washed three times for $10 \mathrm{~min}$ per wash with PBS and incubated with a FITC-conjugated goat antirabbit IgG secondary antibody (Jackson ImmunoResearch, England) for $1 \mathrm{~h}$. All images were visualized by confocal microscopy (LSM 710; Zeiss) and transferred to a computer equipped with Zen Light Edition (Zeiss) for analysis.

\section{miRNA-linked molecular beacon design}

We developed a molecular beacon (MB) to detect miRNA expression in single cells (Kang et al., 2011). We designed a MB for miRNA-23b such that it formed a partially double stranded oligonucleotide. The long oligonucleotide contained the miRNA$23 \mathrm{~b}$ sequence and was the perfect reverse complimentary sequence of mature miRNA-23b (5'-ATCACATTGCCAGGGATTACC- $3^{\prime}$ ) with the sequence -5'-GGTAATCCCTGGCAATGTGAT-3'-CY3. The short oligonucleotide had the sequence Rev-3'- GTTACACTA -5'- BHQ1. The oligonucleotides were manufactured by Bionics (Korea) (Ham et al., 2012).

\section{Luciferase assay}

The predicted gene targets of miRNA-23b were identified using 


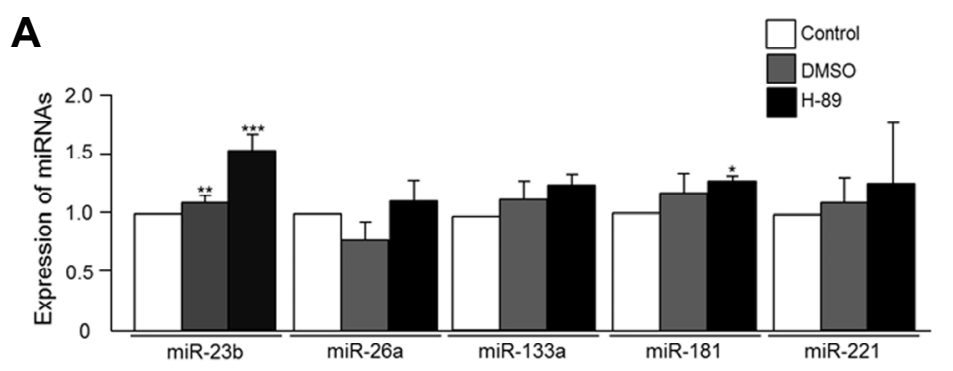

B

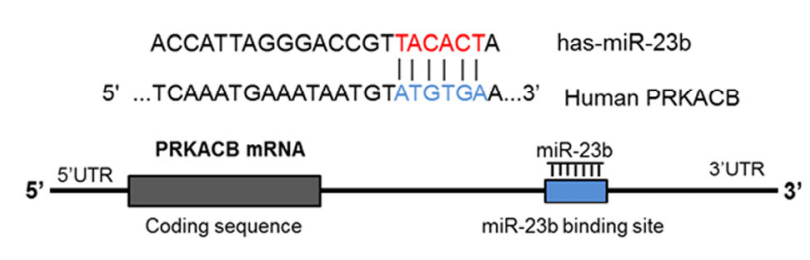

D

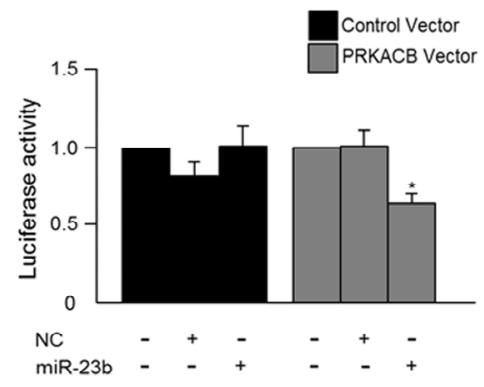

C

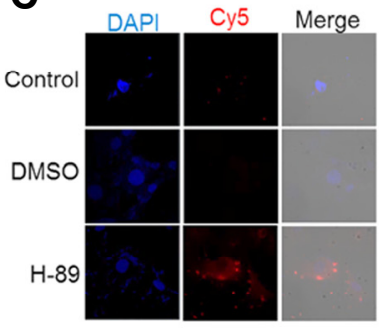

E

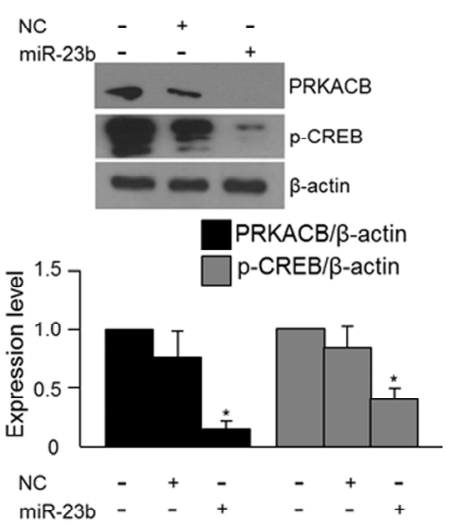

Fig. 2. Expression of miRNA-23b in H-89-treated SFMSCs (A) Expression of various miRNAs was detected by real-time PCR. (B) Structure of the PRKACB 3'UTR showing the binding site of miRNA-23b. (C) Expression of endogenous miRNA-23b in SFMSCs was detected by molecular beacon ICC. Red: miRNA-23b MB. The original magnification for cell images was 400X. The blue color represents DAPI-stained nuclei. (D) PRKACB 3'UTR expression was detected by luciferase assay. Control vector or PRKACB 3'UTR vector was transfected with a negative control (NC) or miRNA-23b mimic. (E) Expression of PRKACB and p-CREB was detected by Western blot ( ${ }^{*} p<0.05$ vs control; ${ }^{* *} p<0.001$ vs control).

a public database (TargetScan, www.targetscan.org). We synthesized the 3'-UTR of PRKACB, which consisted of 3189 base pairs and contained a miRNA-23b binding site. A control sequence containing several mutated bases within the miRNA$23 \mathrm{~b}$ binding site was also synthesized. The corresponding genes were then cloned into the pmirGLO vector. HeLa cells were plated at $2.5 \times 10^{4} /$ well in 24 -well plates. After $48 \mathrm{~h}$, the pmirGLO vector containing the PRKACB binding site for miRNA-23b was co-transfected with miRNA-23b or the negative control using Lipofectamine 2000 (Invitrogen, USA). Renilla luciferase was used to normalize for cell numbers and transfection efficiency. After an additional $48 \mathrm{~h}$, luciferase activity was measured using the Dual Luciferase assay (Promega) according to the manufacturer's instructions (Promega). Each assay was repeated 3 times.

Statistical analysis

Data are expressed as the mean \pm SEM. The significance of the differences between groups was assessed by Student's $t$ test. Comparisons between groups with more than two datasets were performed using one-way ANOVA with Bonferroni correction. P-values less than 0.05 were considered significant.

\section{RESULTS}

Chondrogenic differentiation by inhibiting PKA signaling in SFMSCs

Before the start of the study, we tested the character of SFMSCs compared to BMMSCs and ADMSCs. We detected fibroblast-like morphology and immunophenotype including CD34, CD44, CD45, CD73 (membrane-bound ecto-5'-nucleotisidase), and CD90 (Thy-1). Hematopoietic markers, CD34 and CD45, are not expressed in these cells (BMMSC, ADMSC and SFMSC). However, expression of MSC-positive markers such as CD44, CD73, and CD90 was increased (Chang et al., 2013). We previously showed that treatment of human bone 
A

C

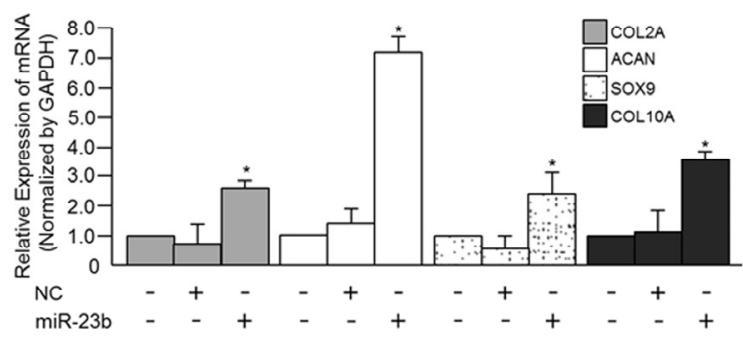

B DAPI Aggrecan Merge

Control

NC

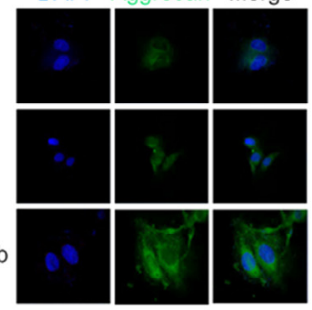

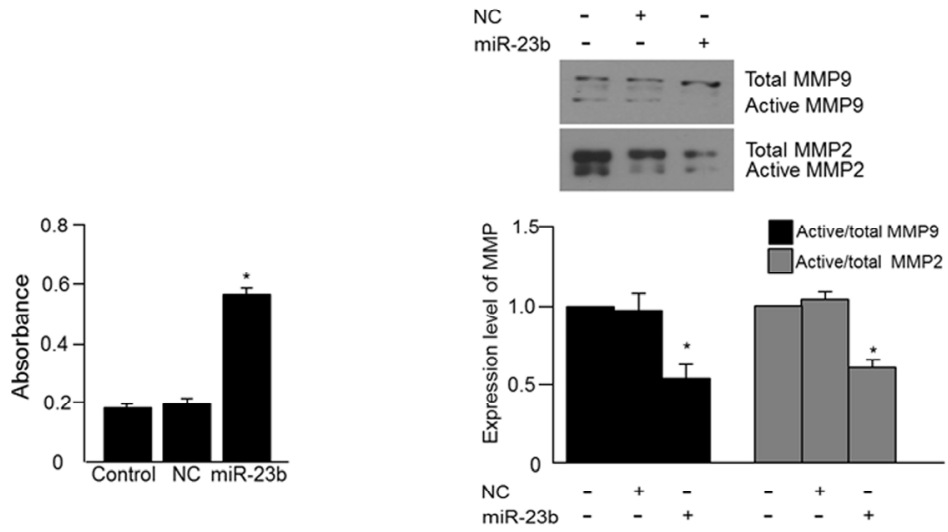

Fig. 3. Effect of miRNA-23b on chondrocyte differentiation. (A) Expression of aggrecan, collagen type II, Sox9, and collagen type X was detected by real-time PCR. (B) Expression of aggrecan was detected by ICC. The original magnification for cell images was 400X. Green, Aggrecan; Blue, DAPI-stained nuclei. (C) Chondrogenic differentiation was measured by alcian blue stain. (D) MMP-9 and MMP-2 were detected by Western blot $\left({ }^{*} \mathrm{p}<0.05\right.$ vs control; ${ }^{* *} \mathrm{p}<0.001$ vs control).

marrow derived MSCs (BMMSCs) with the small molecule protein kinase $\mathrm{A}(\mathrm{PKA})$ inhibitor $\mathrm{H}-89$ results in their differentiation into chondrocytes (Ham et al., 2012). To extend these results, we measured absorbance of alcian blue in SFMSCs and compared it to that of BMMSCs. Increased alcian blue absorbance was detected in SFMSCs compared to BMMSCs treated with $\mathrm{H}-89$ (Fig. 1A). Expression of aggrecan, collagen type II, collagen type $\mathrm{X}$, and Sox9 was also increased in $\mathrm{H}$-89-treated cells (Figs. 1B and $1 \mathrm{C}$ ). As expected, expression of protein kinase $A$ catalytic subunit $B$ (PRKACB) and $p-C R E B$, which are downstream targets of $\mathrm{PKA}$, were down-regulated in $\mathrm{H}-89$ treated cells (Fig. 1D). Because MMPs play a role in matrix remodeling during chondogenic differentiation, we checked their expression. Similarly, treatment with $\mathrm{H}-89$ decreased expression of MMP-9 and MMP-2 (Fig. 1E). $\mathrm{H}-89$ is thought to maintain a cartilage phenotype by inhibiting matrix breakdown by MMPs. Thus, these results suggest that $\mathrm{H}$-89-treated SFMSCs should be able to differentiate into chondrocyte.

\section{Expression of miRNA-23b in H-89-treated cells}

We next evaluated expression of endogenous miRNAs in cells treated with $\mathrm{H}-89$ for 11 days. We previously observed that $\mathrm{H}$ 89 , which was determined to target PKA by Target Scan, induces significant expression of miRNA-23b in chondrocytes (Ham et al., 2012). MiRNA-23b was highly expressed compared to other miRNAs in $\mathrm{H}-89$-treated cells (Fig. 2A). Figure 2B shows the sequence of miRNA-23b and its target PRKACB. Expression of endogenous miRNA-23b was detected at the single cell level using a miRNA-23b molecular beacon (MB) in $\mathrm{H}-89$ treated cells. Increased miRNA-23b level was detected in the cytosol of differentiated chondrogenic cells (Fig. 2C). To determine whether miRNA-23b interacts with the PRKACB 3'UTR, a control or pmirGLO-PRKACB vector was co-transfected along with miRNA-23b or a negative control in HeLa cells. In this assay, targeting the $3^{\prime} U T R$ of PRKACB resulted in decreased luciferase activity (Fig. 2D). Consistent with this observation, the expression of both PRKACB and p-CREB was decreased in miRNA-23b-transfected cells (Fig. 2E). Together, these results indicated that treatment of cells with $\mathrm{H}-89$ induced miRNA23b expression, which inhibited PKA signaling.

\section{Chondrogenic differentiation in miRNA-23b-overexpressed} SFMSCs

We next examined the ability of miRNA-23b to induce chondrogenesis. The expression of the chondrocyte markers collagen type II, collagen type X, and Sox9 was detected by real-time PCR and aggrecan was detected by RT-PCR. All chondrogenic markers were increased in cells transfected with miRNA-23b (Figs. 3A and $3 \mathrm{~B}$ ). In addition, transfection of miRNA-23b resulted in an approximately-3 fold increased in absorbance of alcian blue compared to that of the control (Fig. 3C). As shown in Fig. 1E, transfection with miRNA-23b also resulted in downregulation of MMP-9, and MMP-2 compared to control cells (Fig. $3 \mathrm{D})$. Based on these results, miRNA-23b appeared to induce chondrocyte differentiation. 


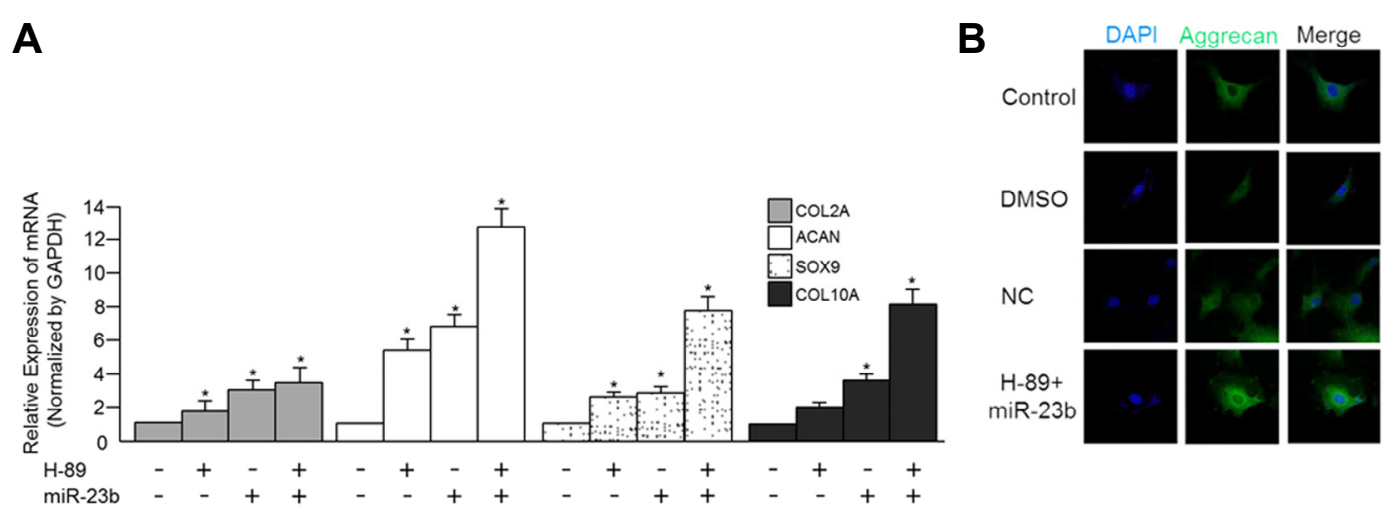

C

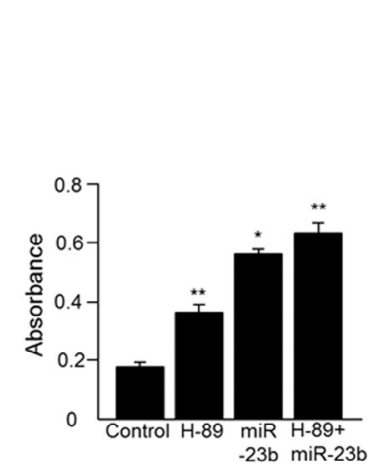

D

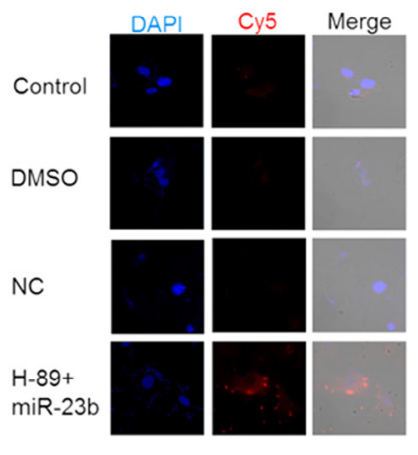

E

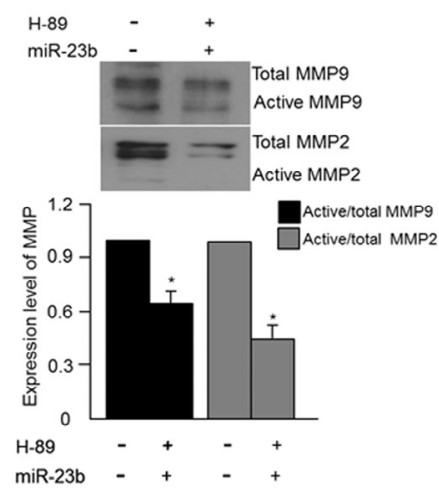

Fig. 4. Chondrogenic differentiation of SFMSCs co-treated with H-89 and miRNA-23b -co-treated. Aggrecan, collagen type II, Sox9, and collagen type X expressions were measured by (A) real-time PCR and (B) ICC. (C) The absorbance of alcian blue was measured at $650 \mathrm{~nm}$. (D) Endogenous miRNA-23b was detected using a miRNA-23b MB (Red). The original magnification for cell images was 400X. All cell images were merged with images of DAPI-stained nuclei. (E) MMP-9, and MMP-2 were detected by Western blot $\left({ }^{*} p<0.05\right.$ vs control; ${ }^{* *} p<0.001$ vs control).

Effects of $\mathrm{H}-89$ and miRNA-23b for differentiation into chondrocyte

We next wanted to determine if co-treatment with miRNA-23b and $\mathrm{H}-89$ could induce differentiation of SFMSCs to chondrocytes. Expression of aggrecan, collagen type II, Sox9 and collagen type $X$ was detected by real-time PCR and intracellular aggrecan level was detected by immunocytochemistry as described above. Cells co-treated with miRNA-23b and $\mathrm{H}-89$ exhibited increased aggrecan expression (Figs. 4A and 4B). Alcian blue staining was increased in cells treated with either $\mathrm{H}-89$ or miRNA-23b, while co-treatment with miRNA-23b and $\mathrm{H}-89$ treated resulted in significantly increased alcian blue staining (Fig. 4C). Endogenous levels of miRNA-23b were also increased in cells co-treated with $\mathrm{H}-89$ and miRNA-23b (Fig. 4D). Lastly, co-treatment of $\mathrm{H}-89$ and miRNA-23b decreased expression of MMP-9 and MMP-2 (Fig. 4E). Together, these data suggest that co-treatment with miRNA-23b and $\mathrm{H}-89$-treated induced chondrogenic differentiation by down-regulating PKA signaling.

\section{DISCUSSION}

MSCs exhibit multipotentiality, and can differentiate into chondrocytes, osteocytes, adipocytes, astrocytes, and cardiac myocytes both in vitro and in vivo. In addition, MSCs possess po- tent immunosuppressive effects that inhibit the activity of both innate and adaptive immune cells, which can improve angiogenesis and prevent fibrosis. Therefore, MSCs are potentially an important source of cells for therapeutic applications for $\mathrm{OA}$ and RA (Lohmander and Roos, 2007).

When joint injury causes rupture and bleeding, synovial fluid contains blood for several days. Such vessel injury and bleeding promotes the expression of cytokines and chemokines including IL-6, TGF- $\beta$, and HGF that consequently recruit MSCs. Inflammation can also increase the number of MSCs during the early healing phase; however, inflammation itself is not sufficient to maintain this increase in MSCs (Morito et al., 2008). Normal healthy SFMSCs are able to differentiate into chondrocytes and can be used for OA treatment (Dunn et al., 2009; Hong and Reddi, 2013). The problems for OA treatment using SFMSCs from normal donors, however, is the required surgical invasion of normal donor, restricted yield of MSCs in synovial fluid (SF) from healthy donor ( $\sim 0.025 \%$ of total SF cells) and limited ex vivo expansion of chondrocytes (Jones et al., 2008; Sekiya et al., 2012). Thus, SFMSCs from patients might be an alternative therapeutic strategy for OA and RA because of the abundance and accessibility of human synovial fluid.

The molecular mechanisms that regulate chondrocyte differentiation are not completely understood. MiRNAs function as endogenous regulators of gene expression by targeting the $3^{\prime}$ 
UTR of target genes. Recently, several studies demonstrated the induction of differentiation of cells into chondrocytes following treatment with miRNAs. Specifically, miRNA-140, -199 and $574-3 p$ have been shown to regulate differentiation of stem cells into chondrocytes (Guérit et al., 2013; Karlsen et al., 2014; Lin et al., 2009). Moreover, various miRNAs including miRNA $140,-143 / 145$ and $-221 /-222$ also regulate chondrogenic differentiation from articular cells (Dunn et al., 2009; Hong and Reddi, 2013). Likewise, protein kinases comprise a large family of proteins that are able to induce differentiation of stem cells into various cell types (Hwang et al., 2008). In this study, we sought to improve the therapeutic possibility of SFMSCs by inducing their differentiation into chondrocytes using miRNA-23b and the small molecule PKA inhibitor $\mathrm{H}-89$.

In previous study, we demonstrated that both miRNA-23b and $\mathrm{H}-89$ induced chondrogenic differentiation in BMMSCs (Ham et al., 2012). Based on our previous research, we studied whether differentiation of SFMSCs into chondrocyte was more useful than differentiation of BMMSCs into chondrocyte for treatment of OA patients via autologous transplantation of differentiated cells. Figure $1 \mathrm{~A}$ showed the increased chondrogenic differentiation potential of SFMSCs from patients compared to BMMSCs. In addition, several studies demonstrated that SFMSCs have the greatest expansion and chondrogenic ability in vitro compared to MSCs from other tissues (Koga et al., 2008; Sakaguchi et al., 2005; Segawa et eal., 2009; Yoshimura et al., 2007). The results indicate that SFMSCs are a superior cell source for treatment of OA or RA patients. Thus, our present study suggested that these differentiated stem cells may be an important tool for autologous stem cell therapy in repairing damaged cartilage.

One problem arising from our study is the expression of type $x$ collagen. To overcome this problem, the method of co-culture, mixing with chondrogenic cells and MSCs, is suggested for the potential cellular crosstalk, instruction, and stabilization of the condrogenic phenotype. This method may be one solution to overcome the problem.

During MSCs differentiation into chondrocyte, MMPs play an important role in terminal endochondral ossification at the hypertrophic stage (Wu et al., 2002). Extracellular matrix (ECM) remodeling by degradation of collagen is also mediated by MMPs (Olivotto et al., 2013). Especially, MMP-1, -2, and -9 play a role for matrix remodeling by inducing degradation of collagens (Lee et al., 2011; Sherriff-Tadano et al., 2006). In our study, expression of MMP-2 and MMP-9 was decreased in chondrogenic differentiated SFMSCs.

Chondrogenic SFMSCs (differentiated cells) are presumed to have several advantages in cell therapy-based cartilage regeneration. These cells can improve callus biomechanical properties and promote cartilage formation. Furthermore, chondrogenic SFMSCs that expressed multiple cartilage-specific like markers can induce formation of stable cartilage as well as contribute to formation of mineralized deposits within the original cartilaginous matrix (Hong and Reddi, 2013; Myers et al., 2010; Pelttari et al., 2008; Vinatier et al., 2009). In addition, transplantation of autologous SFMSCs from patients reduces immunological rejection and ethical controversy.

In conclusion, inhibiting PKA signaling in SFMSCs using $\mathrm{H}-$ 89 and/or miRNA-23b may be a useful tool for developing treatments for patients with degenerative arthritis. Use of chondrogenic differentiated SFMSCs as a unique source of stem cells was an important aspect of this study and may be useful for developing clinically relevant treatments for patients with $R A$ and $\mathrm{OA}$.

\section{ACKNOWLEDGMENTS}

This research was supported by Basic Science Research Program through the National Research Foundation of Korea (NRF) funded by the Ministry of Education, Science and Technology (2013R1A1A1008066), Pusan National University Research Grant, 2013, and a grant (12182MFDS666) from Ministry of Food and Drug safety in 2013, Republic of Korea.

\section{REFERENCES}

Andersen, D.C., Kortesidis, A., Zannettino, A.C., Kratchmarova, I., Chen, L., Jensen, O.N., Teisner, B., Gronthos, S., Jensen, C.H., and Kassem, M. (2011). Development of novel monoclonal antibodies that define differentiation stages of human stromal (mesenchymal) stem cells. Mol. Cells 32, 133-142.

Bagga, S., Bracht, J., Hunter, S., Massirer. K., Holtz, J., Eachus. R., and Pasquinelli, A.E. (2005). Regulation by let-7 and lin-4 miRNAs results in target mRNA degradation. Cell 122, 553-563.

Barry, F.P., and Murphy, J.M. (2004). Mesenchymal stem cells: clinical applications and biological characterization. Int. J. Biochem. Cell. Biol. 36, 568-584

Chang, W., Park, S.I., Jun, S.Y., Lee, E.J., Ham, H.J., Bae, Y.J., Kim R., Park, M.S., Chung, Y.A., Im, N., et al. (2013). Therapeutic potential of autologous mesenchymal stem cells derived from synovial fluid in patients with degenerative arthritis. Animal Cells Syst. 17, 315-324.

Cohen, P. (1999). The development and therapeutic potential of protein kinase inhibitors. Curr. Opin. Chem. Biol. 3, 459-465.

Davies, S.P., Reddy, H., Caivano, M., and Cohen, P. (2000). Specificity and mechanism of action of some commonly used protein kinase inhibitors. Biochem. J. 351, 95-105.

De Ugarte, D.A., Morizono, K., Elbarbary, A., Alfonso, Z., Zuk, P.A., Zhu, M., Dragoo, J.L., Ashjian, P., Thomas, B., Benhaim, P., et al. (2003). Comparison of multi-lineage cells from human adipose tissue and bone marrow. Cells Tissues Organs 174, 101-109.

Dunn, W., DuRaine, G., and Reddi, A.H. (2009). Profiling microRNA expression in bovine articular cartilage and implications for mechanotransduction. Arthritis Rheum. 60, 2333-2339.

Friedenstein, A.J. (1980). Stromal mechanisms of bone marrow: cloning in vitro and retransplantation in vivo. Haematol. Blood Transfus. 25, 19-29.

Giraldez, A.J., Mishima, Y., Rihel, J., Grocock, R.J., Van Dongen, S., Inoue, K., Enright, A.J., and Schier, A.F. (2006). Zebrafish MiR430 promotes deadenylation and clearance of maternal mRNAs. Science 312, 75-79.

Goldring, M.B., Tsuchimochi, K., and ljiri, K. (2006). The control of chondrogenesis. J. Cell. Biochem. 97, 33-44.

Guérit, D., Philipot, D., Chuchana, P., Toupet, K., Brondello, J.M., Mathieu, M., Jorgensen, C., and Noël D. (2013). Sox9-regulated miRNA-574-3p inhibits chondrogenic differentiation of mesenchymal stem cells. PLoS One 8, e62582.

Ham, O., Song, B.W., Lee, S.Y., Choi, E., Cha, M.J., Lee, C.Y., Park J.H., Kim, I.K., Chang, W., Lim, S., et al. (2012). The role of microRNA-23b in the differentiation of MSC into chondrocyte by targeting protein kinase A signaling. Biomaterials 33, 4500-4507.

Hong, E., and Reddi, A.H. (2013). Dedifferentiation and redifferentiation of articular chondrocytes from surface and middle zones: changes in microRNAs-221/-222,-140, and -143/145 expression Tissue Eng. Part A 19, 1015-1022.

Horwitz, E.M., Prockop, D.J., Fitzpatrick, L.A., Koo, W.W., Gordon, P.L., Neel, M., Sussman, M., Orchard, P., Marx, J.C., Pyeritz, R.E., et al. (1999). Transplantability and therapeutic effects of bone marrow-derived mesenchymal cells in children with osteogenesis imperfect. Nat. Med. 5, 309-313.

Hwang, K.C., Kim, J.Y., Chang, W., Kim, D.S., Lim, S., Kang, S.M. Song, B.W., Ha, H.Y., Huh, Y.J., Choi, I.G., et al. (2008) Chemicals that modulate stem cell differentiation. Proc. Natl. Acad. Sci. USA 105, 7467-7471.

Iwata, H., Ono, S., Sato, K., Sato, T., and Kawamura, M. (1993). Bone morphogenetic protein-induced muscle- and synoviumderived cartilage differentiation in vitro. Clin. Orthop. Relat. Res. 296, 295-300.

Jin, H.L., Kim, J.S., Kim, Y.J., Kim, S.J., Broxmeyer, H.E., and Kim, K.S. (2012). Dynamic expression of specific miRNAs during 
erythroid differentiation of human embryonic stem cells. Mol. Cells 34, 177-183.

Jones, E.A., English, A., Henshaw, K., Kinsey, S.E., Markham, A.F., Emery, P., and McGonagle, D. (2004). Enumeration and phenotypic characterization of synovial fluid multipotential mesenchymal progenitor cells in inflammatory and degenerative arthritis. Arthritis Rheum. 50, 817-827.

Jones, E.A., Crawford, A., English, A., Henshaw, K., Mundy, J., Corscadden, D., Chapman, T., Emery, P., Hatton, P., and McGonagle, D. (2008). Synovial fluid mesenchymal stem cells in health and early osteoarthritis: detection and functional evaluation at the single-cell level. Arthritis Rheum. 58, 1731-1740.

Jones, E., Churchman, S.M., English, A., Buch, M.H., Horner, E.A., Burgoyne, C.H., Reece, R., Kinsey, S., Emery, P., and McGonagle, D. (2010). Mesenchymal stem cells in rheumatoid synovium: enumeration and functional assessment in relation to synovial inflammation level. Ann. Rheum. Dis. 69, 450-457.

Karlsen, T.A., Jakobsen, R.B, Mikkelsen, T.S., and Brinchmann, J.E. (2014). microRNA-140 targets RALA and regulates chondrogenic differentiation of human mesenchymal stem cells by translational enhancement of SOX9 and ACAN. Stem Cells Dev. 23, 290-304.

Kang, W.J., Cho, Y.L., Chae, J.R., Lee, J.D., Choi, K.J., and Kim, S. (2011). Molecular beacon-based bioimaging of multiple microRNAs during myogenesis. Biomaterials 32, 1915-1922.

Kobayashi, T., Lu, J., Cobb, B.S., Rodda, S.J., McMahon, A.P., Schipani, E., Merkenschlager, M., and Kronenberg, H.M. (2008). Dicer-dependent pathways regulate chondrocyte proliferation and differentiation. Proc. Natl. Acad. Sci. USA 105, 1949-1954.

Koga, H., Muneta, T., Nagase, T., Nimura, A., Ju, Y.J., Mochizuki, T., and Sekiya, I. (2008). Comparison of mesenchymal tissuesderived stem cells for in vivo chondrogenesis: suitable conditions for cell therapy of cartilage defects in rabbit. Cell Tissue Res. 333, 207-215.

Lee, W.J., Park, S.E., and Rah, D.K. (2011). Effects of hepatocyte growth factor on collagen synthesis and matrix metalloproteinase production in keloids. J. Korean Med. Sci. 26, 1081-1086.

Lee, D.H., Sonn, C.H., Han, S.B., Oh, Y., Lee, K.M., and Lee, S.H. (2012). Synovial fluid CD $34^{-}$CD $44^{+}$CD $90^{+}$mesenchymal stem cell levels are associated with the severity of primary knee osteoarthritis. Osteoarthritis Cartilage 20, 106-109.

Lin, E.A., Kong, L., Bai, X.H., Luan, Y., and Liu, C.J. (2009). miR199a, a bone morphogenic protein 2-responsive MicroRNA, regulates chondrogenesis via direct targeting to Smad1. J. Biol. Chem. 284, 11326-11335.

Lohmander, L.S., and Roos, E.M. (2007). Clinical update: treating osteoarthritis. Lancet 370, 2082-2084.

Morito, T., Muneta, T., Hara, K., Ju, Y.J., Mochizuki, T., Makino, H., Umezawa, A., and Sekiya, I. (2008). Synovial fluid-derived mesenchymal stem cells increase after intra-articular ligament injury in humans. Rheumatology 47, 1137-1143.

Myers, T.J., Granero-Molto, F., Longobardi, L., Li, T., Yan, Y., and Spagnoli, A. (2010). Mesenchymal stem cells at the intersection of cell and gene therapy. Exp. Opin. Biol. Ther. 10, 1663-1679.

Nakamura, Y., Inloes, J.B., Katagiri, T., and Kobayashi, T. (2011). Chondrocyte-specific microRNA-140 regulates endochondral bone development and targets Dnpep to modulate bone morphogenetic protein signaling. Mol. Cell Biol. 31, 3019-3028.

Nishimura, K., Solchaga, L.A., Caplan, A.I., Yoo, J.U., Goldberg, V.M., and Johnstone, B., (1999). Chondroprogenitor cells of synovial tissue. Arthritis Rheum. 42, 2631-2637.

Olivotto, E, Otero, M., Astolfi, A., Platano, D., Facchini, A., Pagani,
S., Flamigni, F., Facchini, A., Goldring, M.B., Borzì, R.M., et al. (2013). IKK $\alpha$ /CHUK regulates extracellular matrix remodeling independent of its kinase activity to facilitate articular chondrocyte differentiation. PLoS One 8, e73024.

Olsen, B.R., Reginato, A.M., and Wang, W. (2000). Bone development. Annu. Rev. Cell Dev. Biol. 16, 191-220.

Pelttari, L., Steck, E., and Richter, W. (2008). The use of mesenchymal stem cells for chondrogenesis. Injury 39, S58-S65.

Prockop, D.J., Azizi, S.A., Colter, D., Digirolamo, C., Kopen, G., and Phinney, D.G. (2000). Potential use of stem cells from bone marrow to repair the extracellular matrix and the central nervous system. Biochem. Soc. Trans. 28, 341-345.

Sakaguchi, Y., Sekiya, I., Yagishita, K., and Muneta, T. (2005). Comparison of human stem cells derived from various mesenchymal tissues: superiority of synovium as a cell source. Arthritis Rheum. 52, 2521-2529.

Segawa, Y., Muneta, T., Makino, H., Nimura, A., Mochizuki, T., Ju, Y.J., Ezura, Y., Umezawa, A., and Sekiya, I. (2009). Mesenchymal stem cells derived from synovium, meniscus, anterior cruciate ligament, and articular chondrocytes share similar gene expression profiles. J. Orthop. Res. 27, 435-441.

Sekiya, I., Ojima, M., Suzuki, S., Yamaga, M., Horie, M., Koga, H., Tsuji, K., Miyaguchi, K., Ogishima, S., Tanaka, H., et al. (2012). Human mesenchymal stem cells in synovial fluid increase in the knee with degenerated cartilage and osteoarthritis. J. Orthop. Res. 30, 943-949.

Sherriff-Tadano, R., Ohta, A., Morito, F., Mitamura, M., Haruta, Y., Koarada, S., Tada, Y., Nagasawa, K., and Ozaki, I. (2006). Antifibrotic effects of hepatocyte growth factor on scleroderma fibroblasts and analysis of its mechanism. Mod. Rheumatol. 16, 364371.

Vinatier, C., Bouffi, C., Merceron, C., Gordeladze, J., Brondello, J.M., Jorgensen, C., Weiss, P., Guicheux, J., and Noël, D. (2009). Cartilage tissue engineering: towards a biomaterial-assisted mesenchymal stem cell therapy. Curr. Stem Cell Res. Ther. 4 318-329.

Wu, C.W., Tchetina, E.V., Mwale, F., Hasty, K., Pidoux, I., Reiner, A., Chen, J., Van Wart, H.E, and Poole, A.R. (2002). Proteolysis involving matrix metalloproteinase 13 (collagenase-3) is required for chondrocyte differentiation that is associated with matrix mineralization. J. Bone Miner Res. 17, 639-651.

Wu, L., Fan, J., and Belasco, J.G. (2006). MicroRNAs direct rapid deadenylation of mRNA. Proc. Natl. Acad. Sci. USA 103, 40344039.

Xiong, X., Kang, X., Zheng, Y., Yue, S., and Zhu, S. (2013). Identification of loop nucleotide polymorphisms affecting microRNA processing and function. Mol. Cells 36, 518-526.

Yamaoka, H., Asato, H., Ogasawara, T., Nishizawa, S., Takahashi, T., Nakatsuka, T., Koshima, I., Nakamura, K., Kawaguchi, H., Chung, U.I., et al. (2006). Cartilage tissue engineering using human auricular chondrocytes embedded in different hydrogel materials. J. Biomed. Mater. Res. 78, 1-11.

Yan, C., Wang, Y., Shen, X.Y., Yang, G., Jian, J., Wang, H.S., Chen, G.Q., and Wu, Q. (2011). MicroRNA regulation associated chondrogenesis of mouse MSCs grown on polyhydroxyalkanoates. Biomaterials 32, 6435-6444.

Yoshimura, H., Muneta, T., Nimura, A., Yokoyama, A., Koga, H., and Sekiya, I. (2007). Comparison of rat mesenchymal stem cells derived from bone marrow, synovium, periosteum, adipose tissue, and muscle. Cell Tissue Res. 327, 449-462.

Zhai, L.J., Zhao, K.Q., Wang, Z.Q., Feng, Y., and Xing, S.C. (2001). Mesenchymal stem cells display different gene expression profiles compared to hyaline and elastic chondrocytes. Int. J. Clin. Exp. Med. 4, 81-90. 